\title{
A DISCRETE KINETIC APPROXIMATION FOR THE INCOMPRESSIBLE NAVIER-STOKES EQUATIONS
}

\author{
Maria Francesca CARfora ${ }^{1}$ And Roberto Natalini ${ }^{2}$
}

\begin{abstract}
In this paper we introduce a new class of numerical schemes for the incompressible NavierStokes equations, which are inspired by the theory of discrete kinetic schemes for compressible fluids. For these approximations it is possible to give a stability condition, based on a discrete velocities version of the Boltzmann H-theorem. Numerical tests are performed to investigate their convergence and accuracy.
\end{abstract}

Mathematics Subject Classification. 65M06, 76M20, 76R.

Received May 24, 2006. Revised April 19, 2007.

\section{Introduction}

In this paper we study finite difference discrete kinetic schemes for the incompressible Navier-Stokes equations:

$$
\left\{\begin{array}{l}
\partial_{t} \mathbf{u}+\operatorname{div}(\mathbf{u} \otimes \mathbf{u})+\nabla \Phi=\nu \Delta \mathbf{u} \\
\operatorname{div} \mathbf{u}=0
\end{array}\right.
$$

with $\left.(x, t) \in \mathbb{R}^{D} \times\right] 0, \infty[$ and with initial data

$$
\mathbf{u}(x, 0)=\mathbf{u}_{0}(x), \quad x \in \mathbb{R}^{D}
$$

Finite difference methods have been widely used because of their relative ease of implementation and flexibility. Starting from the seminal papers by Chorin [10] and Temam [23], many authors have introduced and investigated schemes derived in various frameworks. One of the main problems has been the discretization of the incompressibility condition, which allowed for parasitic modes. Various methods have been proposed to correct the original fractional step method by Chorin, see for instance $[12,13,17,24]$, which however present severe stability limits on the time step. Moreover, the pressure for fractional step methods is only first-order accurate in time, and staggered grids or other numerical corrections are introduced to improve the accuracy.

\footnotetext{
Keywords and phrases. Incompressible fluids, kinetic schemes, BGK models, finite difference schemes.

${ }^{1}$ Istituto per le Applicazioni del Calcolo "M. Picone", Consiglio Nazionale delle Ricerche, Via P. Castellino, 111, 80131, Napoli, Italia. f.carfora@iac.cnr.it

2 Istituto per le Applicazioni del Calcolo "M. Picone", Consiglio Nazionale delle Ricerche, Viale del Policlinico 137, 00161 Roma, Italia. r.natalini@iac.cnr.it
} 
A very interesting alternative approach has been given by Lattice-Boltzmann methods, see for instance [22,25], which employ the evolution of microscopic gas models to approximate macroscopic equations of fluid dynamics. LBMs use discrete velocities models of kinetic equations to obtain approximate solutions to the incompressible Navier-Stokes system, resting on the observation that the two models are equivalent in the limit of small Knudsen and Mach numbers. A few years ago, Junk and Klar [15] presented a discrete velocities model based on a lattice Boltzmann method which leads to a new spatial discretization for the incompressible Navier-Stokes equations, see also [16] for its convergence analysis.

Our approach is inspired on one side by the LBM philosophy and, on the other side, by the kinetic approximations of hydrodynamic equations in the hyperbolic (compressible) setting, see for instance [20] and references therein. As a matter of fact, our main reference is given by the paper by Aregba-Driollet and Natalini [1], where some families of discrete kinetic schemes were introduced for general hyperbolic systems of conservation laws, see also $[8,9]$. These schemes are based on a class of kinetic models with discrete velocities, which convergence has been investigated in [4, 18]. In a more recent paper [2], numerical schemes for nonlinear degenerate parabolic systems with possibly dominant convection have been designed, under a scaling which is similar to the Navier-Stokes's one; general stability conditions have been derived and convergence to the entropy solution has been proved. Discrete kinetic approximations can be also seen as hyperbolic singular perturbations of the Navier-Stokes operator, as investigated in [5,11,19], and from a numerical point of view in [3].

Here we consider a generic BGK model of the form

$$
\partial_{t} f_{l}+\frac{1}{\epsilon} \lambda_{l} \cdot \nabla_{x} f_{l}=\frac{1}{\tau \epsilon^{2}}\left(M_{l}(\rho, \epsilon \rho \mathbf{u})-f_{l}\right), \quad l=1, \ldots, L
$$

with initial condition

$$
f_{l}(x, 0)=M_{l}\left(\bar{\rho}, \epsilon \bar{\rho} \mathbf{u}_{0}\right), \quad l=1, \ldots, L
$$

where $L \geq D+1$; each $f_{l}$ and $M_{l}$ take values in $\mathbb{R}^{D+1}, \epsilon$ is a positive parameter and the $\lambda_{l}=\left(\lambda_{l 1}, \ldots, \lambda_{l D}\right)$ are $\mathbb{R}^{D}$ vectors of fixed constants; the (discrete) Maxwellian functions $M_{l}$ are Lipschitz continuous. Finally, we denote by $f_{l}^{h}, M_{l}^{h}$ for $h=0, \ldots, D$ the $D+1$ components of $f_{l}, M_{l}$, respectively. Then we set

$$
\begin{gathered}
\rho:=\sum_{l=1}^{L} f_{l}^{0}(x, t), \\
\epsilon \rho u_{h}:=\sum_{l=1}^{L} f_{l}^{h}(x, t) .
\end{gathered}
$$

By assuming some suitable consistency conditions on the velocities $\lambda_{l}$ and the Maxwellian functions $M_{l}$, it is possible to show that solutions of this singular perturbation system formally converge to the solutions of the Navier-Stokes equation. In the present paper we study the behavior of some numerical schemes which are derived by this approximation. One of the key features of our approximation is the stability condition obtained via a discrete velocities version of the Boltzmann H-theorem for rarefied gas dynamics, according to the results of [7]. Under this condition it is possible to design effective and stable schemes, with low numerical diffusion.

Even at this early stage it is possible to remark an important difference with standard LBM schemes. In this paper, we work with vector quantities. For every velocity we have one vector of unknowns, which corresponds to the whole solution of the NS equations. This formulation is suitable to prove rigorous stability/entropy results, as we shall see in the following. Similar results, often presented as H-theorems, are sometimes proposed for LBMs, but all the time only for very special situations, see for instance [6]. Concerning the number of velocities, in comparison with standard LBM schemes, we recall that the velocities have to be intended as vector velocities. So, in our case, for every velocity, we have one component for the density and D components for the fluid velocity. So, a five-velocities method in 2 space dimensions corresponds to $(1+2) \times 5=15$ scalar velocities, which are to be compared with standard 9 velocities LBM models. 
The plan of the paper is as follow. In Section 2 we introduce our discrete kinetic models, which in the diffusive scaling are consistent with the Navier-Stokes equations. Some stability conditions are introduced, according to the framework proposed in [7]. Next, a specific model in two space dimensions is introduced, and we are able to set the parameters to fulfil the various stability and consistency conditions. In Section 4, a simple numerical scheme is proposed to approximate the kinetic model, and we give a specific consistency condition at the numerical level. Numerical tests are performed and discussed in the last section.

\section{The DisCRETE KINETIC MODELS}

To construct kinetic models in the form (1.3), we have to choose the velocities $\lambda_{i j}$ and the Maxwellian functions $M_{l}$ to guarantee the consistency with the Navier-Stokes equations. We assume the following compatibility relations to hold:

$$
\begin{array}{ll}
\left(M_{1}\right) \quad & \sum_{i=1}^{L} M_{i}^{0}(\rho, \mathbf{q})=\rho ; \\
\left(M_{2}\right) \quad & \sum_{i=1}^{L} M_{i}^{j}(\rho, \mathbf{q})=q_{j}, \quad j=1, \ldots, D ; \\
\left(M_{3}\right) \quad & \sum_{i=1}^{L} \lambda_{i j} M_{i}^{0}(\rho, \mathbf{q})=q_{j}, \quad j=1, \ldots, D ; \\
\left(M_{4}\right) \quad & \sum_{i=1}^{L} \lambda_{i j} M_{i}^{h}(\rho, \mathbf{q})=\frac{q_{j} q_{h}}{\rho}+P(\rho) \delta_{j h}, \quad j, h=1, \ldots, D ; \\
(V) \quad & \sum_{r, l, k=1}^{D} \sum_{i=1}^{L} \partial_{l k}^{2} \lambda_{i l} \lambda_{i k} \frac{\partial M_{i}^{j}(\bar{\rho}, 0)}{\partial u_{r}} u_{r}=\frac{\nu}{\tau} \sum_{s=1}^{D} \partial_{s}^{2} u_{j}, \quad j=1, \ldots, D ;
\end{array}
$$

where $P(\rho)=\rho^{\gamma}(\gamma \geq 1)$.

\subsection{Consistency of the BGK approximation}

We prove the consistency of the proposed BGK approximation with the incompressible Navier-Stokes equations (1.1) in the limit $\epsilon \rightarrow 0$. To do this, let us state the following preliminary result:

Proposition 2.1. The solution $\mathbf{f}=\left(f_{1}, \ldots, f_{L}\right)$ to the problem (1.3)-(1.4) admits the formal expansion

$$
\mathbf{f}=\mathbf{M}-\epsilon \tau \Lambda \cdot \nabla_{x} \mathbf{M}+\epsilon^{2}\left(-\tau \partial_{t} \mathbf{M}+\tau^{2}\left(\Lambda \cdot \nabla_{x}\right)^{2} \mathbf{M}\right)+O\left(\epsilon^{3}\right) .
$$

Proof. We perform a Chapman-Enksog expansion of $\mathbf{f}$ and obtain

$$
\mathbf{f}=\mathbf{M}-\epsilon^{2} \tau\left(\partial_{t} \mathbf{f}+\frac{\Lambda}{\epsilon} \cdot \nabla_{x} \mathbf{f}\right)=\mathbf{M}+O(\epsilon)
$$

where $\Lambda=\left(\lambda_{1}, \ldots, \lambda_{D}\right)$. Then we have

$$
\begin{aligned}
\mathbf{f} & =\mathbf{M}-\epsilon^{2} \tau\left(\partial_{t} \mathbf{M}+\frac{\Lambda}{\epsilon} \cdot \nabla_{x} \mathbf{M}\right)-\epsilon^{2} \tau\left(\partial_{t}(\mathbf{f}-\mathbf{M})+\frac{\Lambda}{\epsilon} \cdot \nabla_{x}(\mathbf{f}-\mathbf{M})\right) \\
& =\mathbf{M}-\epsilon \tau \Lambda \cdot \nabla_{x} \mathbf{M}+\epsilon^{2}\left(-\tau \partial_{t} \mathbf{M}+\tau^{2} \epsilon^{2} \partial_{t}\left(\partial_{t} \mathbf{f}+\frac{\Lambda}{\epsilon} \cdot \nabla_{x} \mathbf{f}\right)+\tau^{2} \epsilon^{2} \frac{\Lambda}{\epsilon} \cdot \nabla_{x}\left(\partial_{t} \mathbf{f}+\frac{\Lambda}{\epsilon} \cdot \nabla_{x} \mathbf{f}\right)\right) \\
& =\mathbf{M}-\epsilon \tau \Lambda \cdot \nabla_{x} \mathbf{M}+\epsilon^{2}\left(-\tau \partial_{t} \mathbf{M}+\tau^{2}\left(\Lambda \cdot \nabla_{x}\right)^{2} \mathbf{M}\right)+O\left(\epsilon^{3}\right)
\end{aligned}
$$

and the proposition is proved.

We are now able to prove the consistency of our model with (1.1). 
Theorem 2.2. Assume that, under the assumptions $\left(M_{1}\right)-\left(M_{4}\right)$ and $(V)$, the sequence $\left(\rho^{\epsilon}, \mathbf{u}^{\epsilon}\right)$ of solutions to (1.3)-(1.4) converges smoothly, as $\epsilon \rightarrow 0$, to some limit functions $(\tilde{\rho}, \tilde{\mathbf{u}})$. Assume moreover that in some suitable (regular) space,

$$
\frac{P(\rho)-P(\bar{\rho})}{\epsilon^{2}} \rightarrow_{\epsilon \rightarrow 0} \bar{\rho} \mathbf{\Phi},
$$

for some given function $\Phi$, which implies $\tilde{\rho}=\bar{\rho}$. Then the couple $(\tilde{\mathbf{u}}, \mathbf{\Phi})$ is a solution to the incompressible Navier-Stokes equations (1.1).

Proof. Let us consider (1.3) and take the sum over $l$. We obtain

$$
\begin{aligned}
& \partial_{t} \sum_{l=1}^{L} f_{l}^{0}+\sum_{j=1}^{D} \partial_{x_{j}}\left(\sum_{l=1}^{L} \frac{\lambda_{l j}}{\epsilon} f_{l}^{0}\right)=\frac{1}{\tau \epsilon^{2}}\left(\sum_{l=1}^{L} M_{l}^{0}-\sum_{l=1}^{L} f_{l}^{0}\right) \\
& \partial_{t} \sum_{l=1}^{L} f_{l}^{h}+\sum_{j=1}^{D} \partial_{x_{j}}\left(\sum_{l=1}^{L} \frac{\lambda_{l j}}{\epsilon} f_{l}^{h}\right)=\frac{1}{\tau \epsilon^{2}}\left(\sum_{l=1}^{L} M_{l}^{h}-\sum_{l=1}^{L} f_{l}^{h}\right), \quad h=1, \ldots, D .
\end{aligned}
$$

Now, using (1.5) and $\left(M_{1}\right),(2.8)$ gives

$$
\partial_{t} \rho+\sum_{j=1}^{D} \partial_{x_{j}}\left(\sum_{l=1}^{L} \frac{\lambda_{l j}}{\epsilon} f_{l}^{0}\right)=0
$$

whereas, using (1.6) and $\left(M_{2}\right),(2.9)$ gives

$$
\partial_{t}\left(\epsilon \rho u_{h}\right)+\sum_{j=1}^{D} \partial_{x_{j}}\left(\sum_{l=1}^{L} \frac{\lambda_{l j}}{\epsilon} f_{l}^{h}\right)=0, \quad h=1, \ldots, D .
$$

By dividing equation (2.11) by $\epsilon$ and using Proposition 2.1 we have

$$
\begin{aligned}
\partial_{t}\left(\rho u_{h}\right)+\frac{1}{\epsilon} \sum_{j=1}^{D} \partial_{x_{j}}\left(\sum_{l=1}^{L} \frac{\lambda_{l j}}{\epsilon} M_{l}^{h}\right)= & \\
& \frac{\tau}{\epsilon} \sum_{j=1}^{D} \partial_{x_{j}} \sum_{l=1}^{L} \lambda_{l j} \sum_{k=1}^{D} \lambda_{l k} \partial_{x_{k}} M_{l}^{h}+\tau \sum_{j=1}^{D} \partial_{x_{j}} \sum_{l=1}^{L} \lambda_{l j}\left(\partial_{t} M_{l}^{h}-\tau\left(\Lambda \cdot \nabla_{x}\right)^{2} M_{l}^{h}\right)+O(\epsilon) .
\end{aligned}
$$

Using $\left(M_{4}\right)$ and introducing the constant density $\bar{\rho}$, the left hand side of this last equation becomes equal to

$$
\partial_{t}\left(\rho u_{h}\right)+\sum_{j=1}^{D} \partial_{x_{j}}\left(\rho u_{j} u_{h}+\frac{P(\rho)-P(\bar{\rho})}{\epsilon^{2}} \delta_{j h}\right)
$$

Substituting in the right hand side the Taylor expansion for $M$

$$
M(\rho, \epsilon \rho \mathbf{u})=M(\bar{\rho}, \mathbf{0})+\partial_{\rho} M(\bar{\rho}, \mathbf{0})(\rho-\bar{\rho})+\nabla_{q} M(\bar{\rho}, \mathbf{0}) \cdot \epsilon \bar{\rho} \mathbf{u}+O\left(\epsilon^{2}\right)
$$


which is valid in the hypothesis that $\rho-\bar{\rho}$ is at least $O(\epsilon)$, yields, using $(V)$,

$$
\begin{gathered}
\tau \bar{\rho} \sum_{j=1}^{D} \partial_{x_{j}} \sum_{l=1}^{L} \lambda_{l j} \sum_{k=1}^{D} \lambda_{l k} \partial_{x_{k}}\left(\mathbf{u} \cdot \nabla_{q} M_{l}^{h}(\bar{\rho}, \mathbf{0})\right)+O(\epsilon)= \\
\tau \bar{\rho} \sum_{j, k, r} \sum_{l=1}^{L} \lambda_{l j} \lambda_{l k} \partial_{x_{j}} \partial_{x_{k}} \frac{\partial M_{l}^{h}(\bar{\rho}, \mathbf{0})}{\partial u_{r}} u_{r}+O(\epsilon)=\nu \bar{\rho} \sum_{r=1}^{D} \partial_{s}^{2} u_{h}+O(\epsilon) .
\end{gathered}
$$

Then, equating the two sides we finally have

$$
\partial_{t}\left(\rho u_{h}\right)+\sum_{j=1}^{D} \partial_{x_{j}}\left(\rho u_{j} u_{h}+\frac{P(\rho)-P(\bar{\rho})}{\epsilon^{2}} \delta_{j h}\right)=\nu \bar{\rho} \sum_{r=1}^{D} \partial_{s}^{2} u_{h}+O(\epsilon)
$$

Now, from the first conservation law (2.10) and using again the expansion (2.7) for $f$ given by Proposition 2.1, the condition $\left(M_{3}\right)$ and the Taylor expansion for $M$, we obtain

$$
\begin{aligned}
0 & =\partial_{t} \rho+\sum_{j} \partial_{x_{j}} \sum_{i=1}^{L} \frac{\lambda_{i j}}{\epsilon} f_{i}^{0} \\
& =\partial_{t} \rho+\sum_{j=1}^{D} \partial_{x_{j}}\left(\sum_{i=1}^{L} \frac{\lambda_{i j}}{\epsilon}\left(M_{i}^{0}-\epsilon \tau \sum_{k=1}^{D} \lambda_{i k} \partial_{x_{k}} M_{i}^{0}\right)\right)+O\left(\epsilon^{2}\right) \\
& =\partial_{t} \rho+\sum_{j=1}^{D} \partial_{x_{j}}\left(\sum_{i=1}^{L} \frac{\lambda_{i j}}{\epsilon} M_{i}^{0}\right)-\tau \sum_{i=1}^{L} \sum_{j=1}^{D} \sum_{k=1}^{D} \lambda_{i j} \lambda_{i k} \partial_{x_{j}} \partial_{x_{k}} M_{i}^{0}+O\left(\epsilon^{2}\right) \\
& =\partial_{t} \rho+\sum_{j=1}^{D} \partial_{x_{j}}\left(\rho u_{j}\right)+O(\epsilon)
\end{aligned}
$$

Then

$$
\partial_{t} \rho+\sum_{j=1}^{D} \partial_{x_{j}}\left(\rho u_{j}\right)=O(\epsilon)
$$

and the consistency is proved.

Remark 2.3. It is easy to show that in the hyperbolic limit, i.e. when $\tau \rightarrow 0$, while keeping $\epsilon$ fixed, we formally recover the equations:

$$
\left\{\begin{array}{l}
\partial_{t} \rho+\operatorname{div}(\rho \mathbf{u})=0 \\
\partial_{t}(\rho \mathbf{u})+\operatorname{div}(\rho \mathbf{u} \otimes \mathbf{u})+\frac{1}{\epsilon^{2}} \nabla \Phi(\rho)=0 .
\end{array}\right.
$$

This remark is important when we are interested in the construction of kinetic entropies, which are defined by a suitable extension of the reduced entropies for the relaxed system (2.12). It turns out also that in the incompressible limit, as $\epsilon \rightarrow 0$, we finally recover the Incompressible Euler Equations:

$$
\left\{\begin{array}{l}
\partial_{t} \mathbf{u}+\operatorname{div}(\mathbf{u} \otimes \mathbf{u})+\nabla \Phi=0 \\
\operatorname{div} \mathbf{u}=0
\end{array}\right.
$$

However we do not follow this direction in this paper. 


\subsection{Stability conditions: the discrete velocities $\mathbf{H}$-theorem}

Following [7], we introduce some structural conditions to assess entropy inequalities and stability of system (1.3). Consider, as in [1], a general BGK model with a finite set of velocities

$$
\partial_{t} f_{l}+\lambda_{l} \cdot \nabla_{x} f_{l}=\frac{1}{\epsilon}\left(M_{l}(U)-f_{l}\right), \quad l=1, \ldots, L
$$

where, $x \in \mathbb{R}^{D}, t>0, f_{l} \in \mathbb{R}^{K}, \lambda_{l}=\left\{\lambda_{l j}\right\} \in \mathbb{R}^{D}$, and

$$
U=\sum_{l=1}^{L} f_{l} \in \mathbb{R}^{K}
$$

The functions $M_{l}=M_{l}(U)$ are smooth and verify the compatibility condition

$$
\sum_{l=1}^{L} M_{l}(U)=U
$$

Now, set

$$
V_{j}=\sum_{l=1}^{L} \lambda_{l j} f_{l}, \quad F_{j}(U)=\sum_{l=1}^{L} \lambda_{l j} M_{l}(U)
$$

So, we obtain

$$
\left\{\begin{array}{l}
\partial_{t} U+\sum_{j=1}^{D} \partial_{x_{j}} V_{j}=0, \\
\partial_{t} V_{j}+\sum_{l=1}^{L} \sum_{k=1}^{D} \lambda_{l j} \lambda_{l k} \partial_{x_{k}} f_{l}=\frac{1}{\epsilon}\left(F_{j}(U)-V_{j}\right) .
\end{array}\right.
$$

Therefore, in the limit $\epsilon \rightarrow 0$, we obtain the conservation laws

$$
\partial_{t} U+\sum_{j=1}^{D} \partial_{x_{j}} F_{j}(U)=0
$$

In the following, we shall assume implicitly that $U \in \mathcal{U}$, where $\mathcal{U}$ is an open convex subset of $\mathbb{R}^{K}$, and also that $f_{l} \in D_{l}:=\left\{M_{l}(U)\right.$, for $\left.U \in \mathcal{U}\right\}$.

Let $\eta=\eta(U)$ be a standard convex entropy for system (2.18), i.e.: a scalar function such that there exists a set of functions $q_{j}(U)$, the entropy-fluxes, such that:

$$
q_{j}=\nabla \eta^{T} \nabla F_{j}
$$

Kinetic entropies for system (2.14), related to the entropy function $\eta$, are given by convex functions $H_{l}\left(f_{l}\right)$, such that

(1) for every $U \in \mathcal{U}$

$$
\sum_{l=1}^{L} H_{l}\left(M_{l}(U)\right)=\eta(U) ;
$$

(2) for every $f=\left(f_{1}, \ldots, f_{L}\right)$, with $f_{l} \in D_{l}$, let $U_{f}=\sum_{l=1}^{L} f_{l} \in \mathcal{U}$

$$
\sum_{l=1}^{L} H_{l}\left(M_{l}\left(U_{f}\right)\right) \leq \sum_{l=1}^{L} H_{l}\left(f_{l}\right) .
$$


Under these conditions it is easy to deduce a general H-inequality for system (2.14), since

$$
\partial_{t} H_{l}\left(f_{l}\right)+\lambda_{l} \cdot \nabla H_{l}\left(f_{l}\right)=\frac{1}{\epsilon} \nabla_{f} H_{l}\left(f_{l}\right)\left(M_{l}(U)-f_{l}\right) \leq \frac{1}{\epsilon}\left(H_{l}\left(M_{l}\left(U_{f}\right)-H_{l}\left(f_{l}\right)\right)\right) .
$$

Our main tool is given by the results of Bouchut in [7]. First let us introduce the vector space of Maxwellians, associated to a given set of convex entropies $\mathcal{E}$ for system (2.18):

$$
\mathcal{M}^{\mathcal{E}}=\left\{M: \mathcal{U} \rightarrow \mathbb{R}^{L \times K} ; \forall \eta \in \mathcal{E}(\nabla M)^{T} D^{2} \eta \text { symmetric }\right\}
$$

Let us define also the positive convex cone of nonnegative Maxwellians:

$$
\mathcal{M}_{+}^{\mathcal{E}}=\left\{M \in \mathcal{M}^{\mathcal{E}} ; \forall \eta \in \mathcal{E}(\nabla M)^{T} D^{2} \eta \geq 0\right\}
$$

Theorem 2.1 in [7] states that, under some technical conditions, if the Maxwellian $M \in \mathcal{M}_{+}^{\mathcal{E}}$, then there exist kinetic entropies $H_{l}$ for system (2.14), which can be obtained by the relations

$$
\nabla G_{l}=\nabla \eta^{T}(\nabla M), \quad H_{l}\left(M_{l}(U)\right):=G_{l}(U),
$$

and

$$
\nabla H_{l}\left(M_{l}(U)\right) \ni \nabla \eta
$$

in the sense of subdifferentials. In our case, we just use the result by Bouchut using as limit system the Isentropic Euler with entropy function endowed with the strictly convex mechanical entropy:

$$
\eta(\rho, \rho \mathbf{u})=\frac{1}{2} \rho|\mathbf{u}|^{2}+\frac{1}{\gamma} \rho^{\gamma}
$$

So, we obtain

Proposition 2.4. Under the assumptions of Theorem 2.1 in [7], if the Maxwellian $M \in \mathcal{M}_{+}^{\mathcal{E}}$, for smooth solutions to system (1.3)-(1.4) we have the following stability inequality

$$
\int\left[\frac{1}{2} \rho|\mathbf{u}|^{2}+\frac{C}{(\gamma-1) \epsilon^{2}}\left(\rho^{\gamma}-\bar{\rho}^{\gamma}-\gamma \bar{\rho}^{\gamma-1}(\rho-\bar{\rho})\right)\right] \mathrm{d} x+\frac{C}{\epsilon^{4} \tau} \iint|\mathbf{f}-\mathbf{M}|^{2} \mathrm{~d} x \mathrm{~d} t \leq \int \frac{1}{2} \bar{\rho}\left|\mathbf{u}_{0}\right|^{2} \mathrm{~d} x .
$$

Proof. The proof just follows by using the Bouchut kinetic entropy constructed on the mechanical entropy (2.24), which yields:

$$
\partial_{t} \int\left[\mathcal{H}(\mathbf{f})-\mathcal{H}(\overline{\mathbf{M}})-\mathcal{H}^{\prime}(\overline{\mathbf{M}})(\mathbf{f}-\overline{\mathbf{M}})\right] \mathrm{d} x+\frac{1}{\tau \epsilon^{2}} \int\left(\mathcal{H}^{\prime}(\mathbf{f})-\mathcal{H}^{\prime}(\mathbf{M})\right)(\mathbf{f}-\mathbf{M}) \mathrm{d} x=0
$$

To prove that the Maxwellian $M \in \mathcal{M}_{+}^{\mathcal{E}}$, we mainly use the following useful result in [7] (Prop. 2.2).

Proposition 2.5. If $\mathcal{E}$ contains at least one strictly convex entropy, a vector function $M \in \mathcal{M}^{\mathcal{E}}$ is a nonnegative Maxwellian if and only if $\nabla M$ has nonnegative (real) eigenvalues.

Finally let us recall that, a standard asymptotic expansion of the solution of system (2.14) around the equilibrium state, in terms of the singular perturbation parameter $\epsilon$, gives as a second order correction the possibly degenerate parabolic problem:

$$
\partial_{t} u+\sum_{j=1}^{D} \partial_{x_{j}} F_{j}(U)=\sum_{j, k=1}^{D} \partial_{x_{j}}\left[D_{j k}(U) \partial_{x_{k}} U\right]
$$


where $D_{j k}$ is the diffusion of the equation, given by

$$
D_{j k}(U)=\nabla\left(\sum_{l=1}^{L} \lambda_{l j} \lambda_{l k} M_{l}(U)\right)-\nabla F_{j}(U) \nabla F_{k}(U) .
$$

In the following, we shall try to minimize this diffusivity, still keeping this matrix positive definite.

\section{A 5 VELOCITIES MODEL}

To apply our general framework, we need now to fix the set of velocities and the Maxwellian functions. More precisely, we restrict our attention on an OVM model, as in [1]. In such a model, the velocities $\lambda_{i j}$ have to satisfy the two orthogonality conditions

$$
\sum_{i=1}^{L} \lambda_{i j}=0, \quad \sum_{i=1}^{L} \lambda_{i j} \lambda_{i k}=0,
$$

for $j, k=1, \ldots, D$. In the following, we fix $D=2$ for the space dimension. Setting

$$
\begin{gathered}
W=(\rho, \mathbf{q}) \\
A_{1}(W)=\left(q_{1}, \frac{q_{1}^{2}}{\rho}+P(\rho), \frac{q_{1} q_{2}}{\rho}\right) \\
A_{2}(W)=\left(q_{2}, \frac{q_{1} q_{2}}{\rho}, \frac{q_{2}^{2}}{\rho}+P(\rho)\right)
\end{gathered}
$$

we look for the Maxwellian functions in the form

$$
M_{i}(W)=a_{i} W+\sum_{j=1}^{2} b_{i j} A_{j}(W)
$$

where $a_{i}$ and $b_{i j}$ have to satisfy some compatibility conditions derived from conditions $\left(M_{1}\right)-\left(M_{4}\right)$ and $(\mathrm{V})$. Now, let us choose a five velocities model by setting $L=5$ and

$$
c_{1}=(1,0), c_{2}=(0,1), c_{3}=(-1,0), c_{4}=(0,-1), c_{5}=(0,0)
$$

with

$$
\lambda_{i}=\lambda c_{i},
$$

for some $\lambda>0$.

Conditions $\left(M_{1}\right)-\left(M_{4}\right)$ imply

$$
\begin{gathered}
\sum_{i=1}^{L} a_{i}=1, \quad \sum_{i=1}^{L} \lambda_{i j} a_{i}=0, \quad j=1,2 \\
\sum_{i=1}^{L} b_{i j}=0, \quad \sum_{i=1}^{L} \lambda_{i k} b_{i j}=\delta_{j k}, \quad j=1,2 .
\end{gathered}
$$


Then, a possible choice for the coefficients $a_{i}$ and $b_{i j}$ is the following

$$
\begin{aligned}
& a_{1}=\ldots=a_{4}=a, \\
& a_{5}=1-4 a ; \\
b_{11}= & b_{22}=-b_{31}=-b_{42}=\frac{1}{2 \lambda}, \\
b_{i j}= & 0 \text { otherwise. }
\end{aligned}
$$

It is easy to see that these coefficients satisfy conditions (3.32)-(3.33).

To guarantee stability according to Proposition 2.5, diffusivity according to condition (3.40) (that will be given below) and consistency with the viscosity according to condition (V), we need to consider the matrix $\nabla M_{i}$. The following three results hold.

\subsection{Stability}

Proposition 3.1. The eigenvalues $\sigma$ of the gradient of the generic Maxwellian function $M_{i}$, as defined in (3.31), are given by

$$
\sigma_{1}=a_{i}+b_{i 1} q_{1} / \rho+b_{i 2} q_{2} / \rho ; \quad \sigma_{2,3}=\sigma_{1} \pm \sqrt{P^{\prime}(\rho)\left(b_{i 1}^{2}+b_{i 2}^{2}\right)}
$$

Then, a sufficient condition for the stability of the solution to (1.3)-(1.4), using Proposition 2.5 is that, for any index $i$

$$
a_{i}>\sqrt{\left(b_{i 1}^{2}+b_{i 2}^{2}\right) P^{\prime}(\bar{\rho})}
$$

Proof. It is straightforward to see that the generic matrix $\nabla M_{i}$ has the expression

$$
\nabla M_{i}=\left(\begin{array}{ccc}
a_{i} & b_{i 1} & b_{i 2} \\
-b_{i 1} \frac{q_{1}^{2}}{\rho^{2}}-b_{i 2} \frac{q_{1} q_{2}}{\rho^{2}}+b_{i 1} P^{\prime}(\rho) & a_{i}+2 b_{i 1} \frac{q_{1}}{\rho}+b_{i 2} \frac{q_{2}}{\rho} & b_{i 2} \frac{q_{1}}{\rho} \\
-b_{i 1} \frac{q_{1} q_{2}}{\rho^{2}}-b_{i 2} \frac{q_{2}^{2}}{\rho^{2}}+b_{i 2} P^{\prime}(\rho) & b_{i 1} \frac{q_{2}}{\rho} & a_{i}+b_{i 1} \frac{q_{1}}{\rho}+2 b_{i 2} \frac{q_{2}}{\rho}
\end{array}\right) .
$$

Then, its characteristic equation is

$$
\left(a_{i}-\sigma+b_{i 1} \frac{q_{1}}{\rho}+b_{i 2} \frac{q_{2}}{\rho}\right)^{3}-P^{\prime}(\bar{\rho})\left(b_{i 1}^{2}+b_{i 2}^{2}\right)\left(a_{i}-\sigma+b_{i 1} \frac{q_{1}}{\rho}+b_{i 2} \frac{q_{2}}{\rho}\right)=0
$$

and the theorem holds.

If the coefficients $a_{i}$ and $b_{i j}$ are chosen as in (3.34)-(3.35), condition (3.36) reads, setting $P^{\prime}(\bar{\rho})=m^{2}$,

$$
\frac{1}{4}>a>\frac{m}{2 \lambda},
$$

and implies

$$
\lambda>2 m \text {. }
$$




\subsection{Diffusivity}

According to (2.27), let the diffusion tensor be given by

$$
D_{j k}=\sum_{l=1}^{L} \lambda_{l j} \lambda_{l k} \nabla M_{l}-\nabla A_{j} \nabla A_{k}
$$

and let us recall that a kinetic model is strictly diffusive if, $\forall \xi \in \mathbb{R}^{D} \backslash\{0\}$

$$
D(\xi):=\sum_{l k} D_{l k}(\bar{\rho}, 0) \xi_{l} \xi_{k} \text { is invertible. }
$$

Proposition 3.2. The diffusion tensor for the five velocities model defined by (3.31)-(3.29)-(3.30) is given by

$$
D(\xi)=\left(\begin{array}{ccc}
\left(2 \lambda^{2} a-P^{\prime}(\bar{\rho})\right)|\xi|^{2} & 0 & 0 \\
0 & \left(2 \lambda^{2} a\right)|\xi|^{2}-P^{\prime}(\bar{\rho}) \xi_{1}^{2} & -P^{\prime}(\bar{\rho}) \xi_{1} \xi_{2} \\
0 & -P^{\prime}(\bar{\rho}) \xi_{1} \xi_{2} & \left(2 \lambda^{2} a\right)|\xi|^{2}-P^{\prime}(\bar{\rho}) \xi_{2}^{2}
\end{array}\right) .
$$

Then, in the hypothesis of stability, the model is also strictly diffusive.

Proof. It is

$$
\begin{aligned}
& \nabla M_{i}(\bar{\rho}, \mathbf{0})=\left(\begin{array}{ccc}
a_{i} & b_{i 1} & b_{i 2} \\
b_{i 1} P^{\prime}(\bar{\rho}) & 0 & 0 \\
b_{i 2} P^{\prime}(\bar{\rho}) & 0 & 0
\end{array}\right) \\
& \nabla A_{1}(\bar{\rho}, \mathbf{0})=\left(\begin{array}{ccc}
0 & 1 & 0 \\
P^{\prime}(\bar{\rho}) & 0 & 0 \\
0 & 0 & 0
\end{array}\right) \\
& \nabla A_{2}(\bar{\rho}, \mathbf{0})=\left(\begin{array}{ccc}
0 & 0 & 1 \\
0 & 0 & 0 \\
P^{\prime}(\bar{\rho}) & 0 & 0
\end{array}\right) .
\end{aligned}
$$

Therefore, the diffusion tensor has the expression (3.41) and condition (3.40) reads

$$
\operatorname{det}(D(\xi))=\left(2 \lambda^{2} a-P^{\prime}(\bar{\rho})\right)^{2} 2 \lambda^{2} a|\xi|^{6} \neq 0,
$$

which is verified (actually $>0$ ) for $a>\frac{P^{\prime}(\bar{\rho})}{2 \lambda^{2}}$. Now, assuming (3.38), this condition holds true without any further condition. 


\subsection{Consistency with the viscosity}

As shown in (3.37), we have the equality

$$
\frac{\partial M_{i}^{j}}{\partial q_{r}}(\bar{\rho}, \mathbf{0})=a_{i} \delta_{j r}
$$

where $\mathbf{q}$ stands for $\epsilon \rho \mathbf{u}$, so that the left hand side of condition $(V)$ reads

$$
\sum_{r, l, k=1}^{D} \sum_{i=1}^{L} \partial_{l k}^{2} \lambda_{i l} \lambda_{i k} \frac{\partial M_{i}^{j}(\bar{\rho}, \mathbf{0})}{\partial q_{r}} q_{r}=\epsilon \bar{\rho} \sum_{l, k=1}^{D} \partial_{l k}^{2} u_{j} \sum_{i=1}^{L} a_{i} \lambda_{i l} \lambda_{i k}=2 a \epsilon \lambda^{2} \bar{\rho} \sum_{k=1}^{D} \partial_{k k}^{2} u_{j}
$$

where the orthogonality of the $\lambda_{i j}$ has been used.

The condition $(V)$ becomes in our case:

$$
2 a \epsilon \lambda^{2} \bar{\rho} \sum_{k=1}^{D} \partial_{k k}^{2} u_{j}=\epsilon \bar{\rho} \frac{\nu}{\tau} \sum_{s=1}^{D} \partial_{s s}^{2} u_{j}
$$

and the condition for consistency is given by

$$
\tau=\frac{\nu}{2 \lambda^{2} a}
$$

\section{NUMERICAL SCHEMES}

In this section we use the kinetic model introduced in Section 3 to design a numerical scheme for the NavierStokes equations (1.1). The general idea is to construct a discretization of (1.3) in order to obtain, for sufficiently small $\epsilon$, a scheme for (1.1). As reported in the previous section, the Maxwellian functions are defined by (3.31); the constant density $\bar{\rho}$ is fixes equal to one.

We use a kinetic splitting and we connect $\epsilon$ with the discretization parameter $\Delta x$ so that this process provides consistent approximations of (1.1) (as will be proved in Sect. 4.1). Let us first introduce some notations. In this paper we restrict ourselves to cartesian uniform grids:

$$
\mathbb{R}^{D}=\bigcup_{\alpha \in \mathbb{Z}^{D}} \mathrm{I}_{\alpha}, \quad[0, T]=\bigcup_{0 \leq n \leq N-1}\left[t_{n}, t_{n+1}\right]
$$

Set $\alpha=\left(\alpha_{d}\right)_{1 \leq d \leq D} \in \mathbb{Z}^{D}$ and let $e_{d}$ be the canonical $d^{\text {th }}$ vector in $\mathbb{R}^{D}$. As usual we denote by $x_{\alpha}$ the center of $\mathrm{I}_{\alpha}, \Delta x_{d}$ the length of $\mathrm{I}_{\alpha}$ in the direction $d, \Delta t=t_{n+1}-t_{n}, \Delta x=\left(\Delta x_{d}\right)_{1 \leq d \leq D}$. Finally we set

$$
\begin{gathered}
f_{\Delta}^{\epsilon}(x, t)=\sum_{n=0}^{N-1} \sum_{\alpha \in \mathbb{Z}^{D}} f_{\alpha}^{\epsilon, n} \mathbf{I}_{\mathbf{I}_{\alpha}}(x) \mathbf{I}_{] t_{n}, t_{n+1}[}(t), \\
f_{\Delta}^{\epsilon, n}(x)=\sum_{\alpha \in \mathbb{Z}^{D}} f_{\alpha}^{\epsilon, n} \mathbf{I}_{\mathrm{I}_{\alpha}}(x), \\
f^{\epsilon, n}=\left(f_{\alpha}^{\epsilon, n}\right)_{\alpha \in \mathbb{Z}^{D}},
\end{gathered}
$$

where any of these functions take values in $\mathbb{R}_{L}$. If

$$
u_{\alpha}^{0}=\left(\operatorname{vol}\left(\mathrm{I}_{\alpha}\right)\right)^{-1} \int_{\mathrm{I}_{\alpha}} u_{0}(x) \mathrm{d} x
$$


then the initial datum $f_{0}^{\epsilon}$ is approximated by

$$
f_{\alpha}^{\epsilon, 0}=M\left(u_{\alpha}^{0}\right)
$$

We split equation (1.3) into its hyperbolic diagonal linear part and an ordinary differential system: for a given $f_{\Delta}^{\epsilon, n}$, the function $f_{\Delta}^{\epsilon, n+1 / 2}$ is an approximate solution at time $t_{n+1}$ to the problem

$$
\begin{gathered}
\partial_{t} f+\frac{1}{\epsilon} \Lambda \cdot \nabla_{x} f=0, \\
f\left(t_{n}\right)=f_{\Delta}^{\epsilon, n} .
\end{gathered}
$$

As the system is diagonal, we may consider each equation separately. We suppose that the scheme is stable and can be put in conservation form:

$$
f_{\alpha, l}^{\epsilon, n+1 / 2}=f_{\alpha, l}^{\epsilon, n}-\sum_{j=1}^{D} \frac{\lambda_{l j}}{\epsilon} \frac{\Delta t}{\Delta x_{j}}\left(f_{\alpha+\frac{1}{2}, l}^{\epsilon, n}-f_{\alpha-\frac{1}{2}, l}^{\epsilon, n}\right), \quad l=1, \ldots, L .
$$

As a particular case we use a three points monotone schemes with standard CFL conditions, see [3] and below.

In the following, the scheme on the linear part will be referred to as (HS) (homogeneous scheme) and the associated evolution operator will be denoted by $H_{\Delta}^{\epsilon}$ :

$$
f_{\Delta}^{\epsilon, n+1 / 2}=H_{\Delta}^{\epsilon}(\Delta t) f_{\Delta}^{\epsilon, n} .
$$

From this approximate solution we find by (1.5)-(1.6) the related values $\rho_{\Delta}^{\epsilon, n+1}, \mathbf{u}_{\Delta}^{\epsilon, n+1}$ of the macroscopic variables.

Now, to take into account the contribution of the term on the right-hand side of (1.3), as a second step of this fractional step method we solve analytically the ordinary differential equation

$$
\partial_{t} f=\frac{1}{\tau \epsilon^{2}}(M(\rho, \mathbf{q})-f)
$$

with the initial condition

$$
f\left(t_{n}\right)=f_{\Delta}^{\epsilon, n+1 / 2}
$$

obtaining

$$
f_{\Delta}^{\epsilon, n+1}=M\left(\rho\left(t_{n+1}\right), \mathbf{q}\left(t_{n+1}\right)\right)+\exp \left(-\frac{\Delta t}{\tau \epsilon^{2}}\right)\left(f_{\Delta}^{\epsilon, n+1 / 2}-M\left(\rho\left(t_{n+1}\right), \mathbf{q}\left(t_{n+1}\right)\right)\right)
$$

where $\tau$ has to be chosen from (3.42) to satisfy consistency with the viscosity.

As an alternative, we can project $f_{\Delta}^{\epsilon, n+1 / 2}$ on the equilibrium distribution, obtaining a different solution

$$
f_{\Delta, p r o j}^{\epsilon, n+1}=M\left(\rho_{\Delta}^{\epsilon, n+1}, \rho \mathbf{u}_{\Delta}^{\epsilon, n+1}\right) .
$$

\subsection{Consistency of the numerical approximation}

In this section we study the consistency of the numerical approximation when the solution to the homogeneous problem (4.47) is simply projected into the equilibrium distribution as in (4.51).

In the following we fix $D=2$ and take $\Delta x=\Delta y=h$ and $\Delta t=k$. Apply to the homogeneous problem

$$
\partial_{t} f^{\epsilon}+\frac{1}{\epsilon} \lambda_{j} \cdot \nabla_{x} f^{\epsilon}=0
$$


the multidimensional upwind scheme, which is a particular case of the scheme (4.47). We analyze its consistency by using standard Taylor expansion. For our orthogonal model we find, using the condition $\lambda_{i 1} \lambda_{i 2}=0$ and since $\left|\lambda_{i j}\right|=0$ or $\lambda$ :

$$
\begin{gathered}
\partial_{t} f_{1}+\frac{\lambda}{\epsilon} \partial_{x} f_{1}=\frac{\lambda}{2 \epsilon}\left(h-\frac{\lambda}{\epsilon} k\right) \partial_{x x} f_{1}, \\
\partial_{t} f_{2}+\frac{\lambda}{\epsilon} \partial_{y} f_{2}=\frac{\lambda}{2 \epsilon}\left(h-\frac{\lambda}{\epsilon} k\right) \partial_{y y} f_{2}, \\
\partial_{t} f_{3}-\frac{\lambda}{\epsilon} \partial_{x} f_{3}=\frac{\lambda}{2 \epsilon}\left(h-\frac{\lambda}{\epsilon} k\right) \partial_{x x} f_{3}, \\
\partial_{t} f_{4}-\frac{\lambda}{\epsilon} \partial_{y} f_{4}=\frac{\lambda}{2 \epsilon}\left(h-\frac{\lambda}{\epsilon} k\right) \partial_{y y} f_{4}, \\
\partial_{t} f_{5}=0 .
\end{gathered}
$$

Let us fix now

$$
\epsilon=\xi h, k=\mu \frac{\epsilon h}{\lambda}(0<\mu<1) .
$$

Therefore

$$
\frac{\lambda}{2 \epsilon}\left(h-\frac{\lambda}{\epsilon} k\right)=\frac{\lambda}{2 \xi}(1-\mu)
$$

which is constant, and we have to proceed with a careful investigation of the behavior of the second derivatives. Let us recall now that in the transport-projection method we update the $f$ at time $t_{n+1}$ according to equation (4.51). By summing up on $i$, we find

$$
\partial_{t}\left(\sum_{i} M_{i}\right)+\sum_{j} \partial_{j}\left(\sum_{i} \frac{\lambda_{i j}}{\epsilon} M_{i}\right)=\frac{\lambda}{2 \epsilon}\left(h-\frac{\lambda}{\epsilon} k\right)\left(\partial_{x x}\left(M_{1}+M_{3}\right)+\partial_{y y}\left(M_{2}+M_{4}\right)\right) .
$$

Recall that, by definition (3.31)

$$
\begin{aligned}
& M_{i}^{0}=a_{i} \rho+b_{i 1} \epsilon \rho u_{1}+b_{i 2} \epsilon \rho u_{2}=a_{i} \bar{\rho}+O(\epsilon) \\
& M_{i}^{1}=a_{i} \epsilon \rho u_{1}+b_{i 1}\left(\epsilon^{2} \rho u_{1}^{2}+P(\rho)\right)+b_{i 2}\left(\epsilon^{2} \rho u_{1} u_{2}\right)=a_{i} \epsilon \bar{\rho} u_{1}+O\left(\epsilon^{2}\right) \\
& M_{i}^{2}=a_{i} \epsilon \rho u_{2}+b_{i 1}\left(\epsilon^{2} \rho u_{1} u_{2}\right)+b_{i 2}\left(\epsilon^{2} \rho u_{2}^{2}+P(\rho)\right)=a_{i} \epsilon \bar{\rho} u_{2}+O\left(\epsilon^{2}\right) .
\end{aligned}
$$

Now, using conditions (3.32)-(3.33), we have

$$
\partial_{t} \rho+\sum_{j} \partial_{j}\left(\rho u_{j}\right)=\frac{\lambda}{2 \xi}(1-\mu)\left(\partial_{x x}\left(M_{1}^{0}+M_{3}^{0}\right)+\partial_{y y}\left(M_{2}^{0}+M_{4}^{0}\right)\right)
$$

which gives the consistency immediately. In the same way we have

$$
\partial_{t}\left(\rho u_{l}\right)+\sum_{j} \partial_{j}\left(\rho u_{j} u_{l}+\frac{P(\rho)-P(\bar{\rho})}{\epsilon^{2}} \delta_{j l}\right)=\frac{\lambda}{2 \epsilon \xi}(1-\mu)\left(\partial_{x x}\left(M_{1}^{l}+M_{3}^{l}\right)+\partial_{y y}\left(M_{2}^{l}+M_{4}^{l}\right)\right) .
$$

We have that

$$
\begin{aligned}
& \partial_{x x}\left(M_{1}^{l}+M_{3}^{l}\right)=\epsilon\left(a_{1}+a_{3}\right) \bar{\rho} \partial_{x x} u^{l}+O\left(\epsilon^{2}\right), \\
& \partial_{y y}\left(M_{2}^{l}+M_{4}^{l}\right)=\epsilon\left(a_{2}+a_{4}\right) \bar{\rho} \partial_{y y} u^{l}+O\left(\epsilon^{2}\right) .
\end{aligned}
$$


TABLE $1 . L_{1}$ and $L_{\infty}$ relative errors on the retrieved velocity components and pressure at increasing resolution for Test case 1. Estimated rates of convergence are also reported.

\begin{tabular}{c|ccc|ccc|ccc} 
& \multicolumn{4}{|c|}{$L_{1}$} & \multicolumn{3}{|c|}{ Rate } & \multicolumn{3}{c}{$L_{\infty}$} \\
\hline$n x$ & $u_{1}$ & $u_{2}$ & $p$ & $u_{1}$ & $u_{2}$ & $p$ & $u_{1}$ & $u_{2}$ & $p$ \\
\hline 32 & 0.27990 & 0.18706 & 0.32548 & - & - & - & 0.31851 & 0.19473 & 0.36339 \\
64 & 0.08460 & 0.04549 & 0.16930 & 1.7 & 2.1 & 1.0 & 0.07665 & 0.04832 & 0.20380 \\
128 & 0.02374 & 0.01035 & 0.07107 & 1.8 & 2.2 & 1.2 & 0.02161 & 0.00973 & 0.10491 \\
256 & 0.00557 & 0.00320 & 0.05822 & 2.1 & 1.6 & 0.6 & 0.00486 & 0.00271 & 0.07670 \\
512 & 0.00088 & 0.00043 & 0.06907 & 3.1 & 3.7 & 0.4 & 0.00080 & 0.00036 & 0.09492
\end{tabular}

This yields

$$
\partial_{t}\left(\rho u_{l}\right)+\sum_{j} \partial_{j}\left(\rho u_{j} u_{l}+\frac{P(\rho)-P(\bar{\rho})}{\epsilon^{2}} \delta_{j l}\right)=\frac{\lambda a}{\xi}(1-\mu) \bar{\rho} \Delta u_{l} .
$$

Therefore, to obtain the consistency with (1.1), we have to choose

$$
a=\frac{\xi \nu}{\lambda(1-\mu) \bar{\rho}} .
$$

Set $\beta=\frac{\xi}{1-\mu}$. Then our conditions read now

$$
\lambda>2 m, \quad \frac{\lambda}{4 \nu}>\beta>\frac{m}{2 \nu} .
$$

Finally, a complete set of conditions is given by

$$
\mu=\frac{1}{2}, \quad m=1, \quad a=\frac{\beta \nu}{\lambda}, \quad \lambda>2, \quad \frac{\lambda}{4 \nu}>\beta>\frac{1}{2 \nu} .
$$

Let us force the system to the minimal viscosity, by taking

$$
\beta=\frac{1}{2 \nu}, \quad \lambda=2, \quad a=\frac{1}{4} .
$$

In this case $a_{5}=0$ and we can neglect the zero speed. Finally, in the scheme we take

$$
\epsilon=\frac{1}{4 \nu} h \bar{\rho}, k=\frac{h^{2}}{16 \nu} .
$$

\section{Numerical RESUlts}

In this section we assess the performance of the proposed BGK approximation on some typical test problems. We choose such problems since they have analytical solution and constitute a quite common benchmark for numerical schemes in the literature. We apply the kinetic splitting to the 5 -velocities orthogonal model introduced in Section 3: the hyperbolic linear system (4.45) is discretized by finite differences and solved by a first order upwind scheme; then each $f_{i}$ is projected to the equilibrium state.

We apply explicit time discretization schemes to the advection part of the scheme. Indeed, the CPU time required to run the considered test problems on a Pentium IV workstation is of the order of seconds/minutes. It is possible to argue that implicit schemes could allow for larger time steps, probably resulting in some reduction of the computational cost; however, the minor accuracy of implicit schemes for high time steps and the significant computational effort they require on very fine resolution grids motivated us not to use them. 
TABLE 2. Test case 2; Reynolds $=100$; pressure drop $\Delta \Phi=0.12 . L_{1}$ and $L_{\infty}$ absolute errors on the retrieved velocity components and pressure at increasing resolution. Estimated rates of convergence are also reported.

\begin{tabular}{c|ccc|ccc|ccc} 
& \multicolumn{4}{|c|}{$L_{1}$} & \multicolumn{3}{|c|}{ Rate } & \multicolumn{3}{c}{$L_{\infty}$} \\
\hline$n x$ & $u_{1}$ & $u_{2}$ & $p$ & $u_{1}$ & $u_{2}$ & $p$ & $u_{1}$ & $u_{2}$ & $p$ \\
\hline 40 & 0.03169 & 0.02421 & 0.03405 & - & - & - & 0.08295 & 0.07982 & 0.06323 \\
80 & 0.00871 & 0.00716 & 0.01574 & 1.8 & 1.7 & 1.1 & 0.02710 & 0.01452 & 0.04412 \\
160 & 0.00233 & 0.00213 & 0.01033 & 1.8 & 1.7 & 0.8 & 0.00478 & 0.00415 & 0.01879 \\
320 & 0.00113 & 0.00059 & 0.00557 & 1.1 & 1.8 & 1.0 & 0.00185 & 0.00146 & 0.01223
\end{tabular}

The first test problem we have considered is the evolution of a decaying Taylor vortex in a $2 \pi$ periodic domain, as in [21]. The exact solution for the flow satisfies

$$
\begin{aligned}
& u_{1}(x, y, t)=-\exp \left(-\nu t\left(w_{1}^{2}+w_{2}^{2}\right)\right) \cos \left(w_{1} x\right) \sin \left(w_{2} y\right) \\
& u_{2}(x, y, t)=\frac{w_{1}}{w_{2}} \exp \left(-\nu t\left(w_{1}^{2}+w_{2}^{2}\right)\right) \sin \left(w_{1} x\right) \cos \left(w_{2} y\right)
\end{aligned}
$$

while, up to a constant, the pressure is

$$
\Phi(x, y, t)=-0.25 \exp \left(-2 \nu t\left(w_{1}^{2}+w_{2}^{2}\right)\right)\left(\cos \left(2 w_{1} x\right)+\frac{w_{1}^{2}}{w_{2}^{2}} \cos \left(2 w_{2} y\right)\right)
$$

We fix the Reynolds number $R e=100$ and two different space parameters in the $x$ and $y$ directions, $w_{1}=3$ and $w_{2}=2$, to avoid symmetry. We solve the NS equations on the square $[0,2 \pi]^{2}$ with $n x$ nodes on each side of the square. The maximal time for computation is set to $T=1$.

Table 1 shows accuracy results of the numerical method: the relative errors on the velocity components and on the pressure at increasing resolution in discrete $L_{1}$ and $L_{\infty}$ norms are reported. Moreover, convergence rates are also evaluated as

$$
\log _{2}\left(\frac{e_{k}}{e_{k+1}}\right)
$$

where $e$ indicates the $L_{1}$ error and subscripts $k, k+1$ refer as usual to two subsequent resolutions. Let us note that the convergence rate for $u_{1}$ and $u_{2}$ is about two, while for the pressure we obtain only a slower convergence (almost first order).

Beside of these global accuracy results, we consider in more details the convergence history at local maxima and minima of the flow. Figure 1 shows the graphic representation of the cross section of the exact solution where the vortex attain their maxima and minima $\left(y=\pi\right.$ for the $u_{1}$ component, $x=\pi$ for the $u_{2}$ component) and compares it with the computed ones at four different resolutions; Figure 2 shows the same representation for the cross section where the exact solution vanishes $\left(x=\pi\right.$ for the $u_{1}$ component, $y=\pi$ for the $u_{2}$ component); while, as can be seen in the first double figure, the peaks are well fitted even at a medium resolution, in this second figure we note that the effects of the increasing resolution are more pronounced.

The second test problem is a Poiseuille flow as in [14]. Along an infinitely long channel of unitary width a pressure gradient is applied. It gives rise to a stationary flow with a parabolic velocity profile which is stable for low Reynolds numbers. If the channel stretches in $x$ direction and the pressure drop is $\Delta p$ on an $x$ interval of unitary length then the solution has the form

$$
\begin{aligned}
& u_{1}=\frac{\Delta p}{2 \rho \nu} y(1-y) \\
& u_{2}=0
\end{aligned}
$$



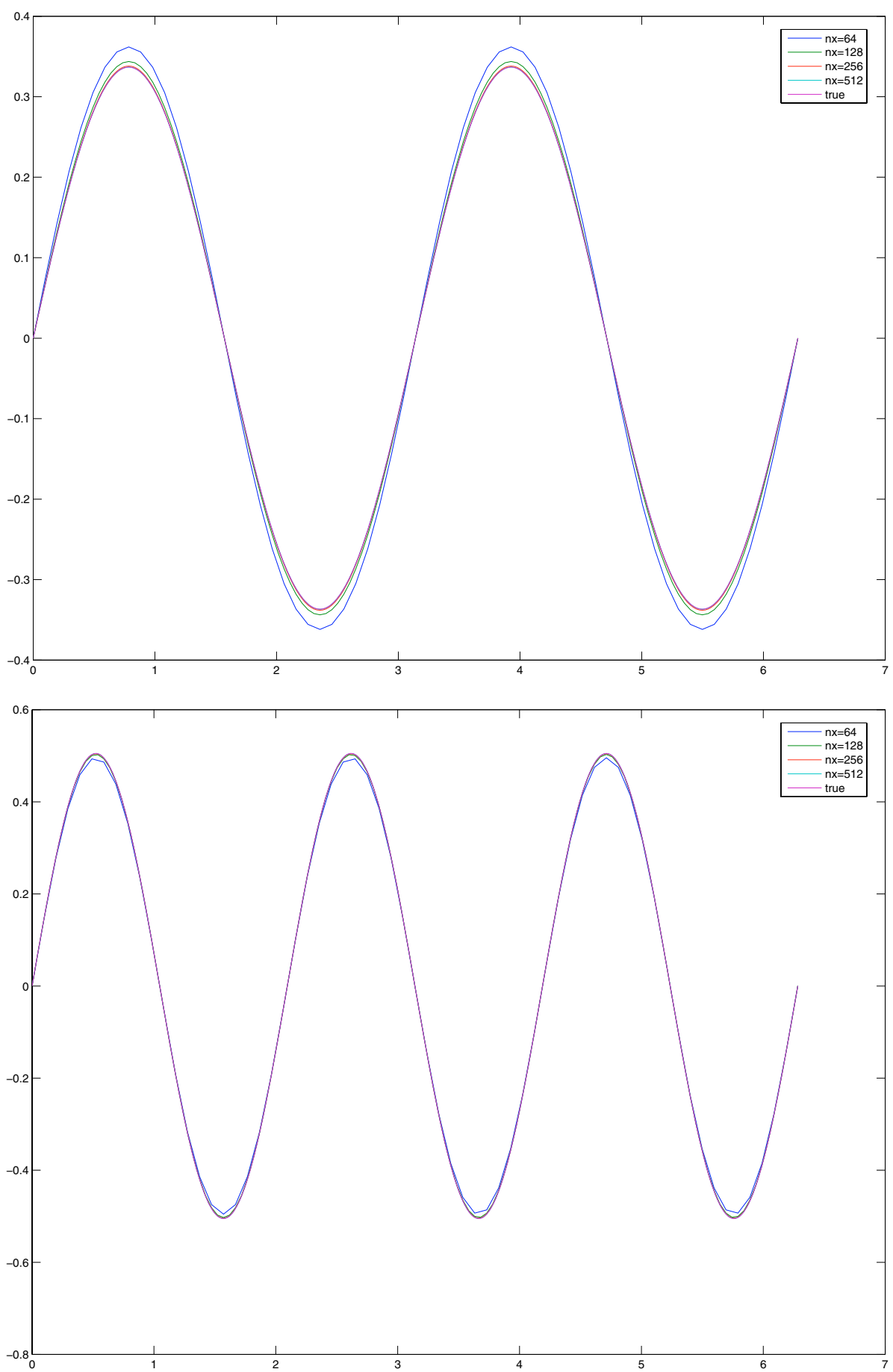

Figure 1. Cross-section of the computed and exact solution: on the top the $u_{1}$ component for $y=\pi$, on the bottom the $u_{2}$ component for $x=\pi$. 
A KINETIC APPROXIMATION FOR THE NAVIER-STOKES EQUATIONS

109
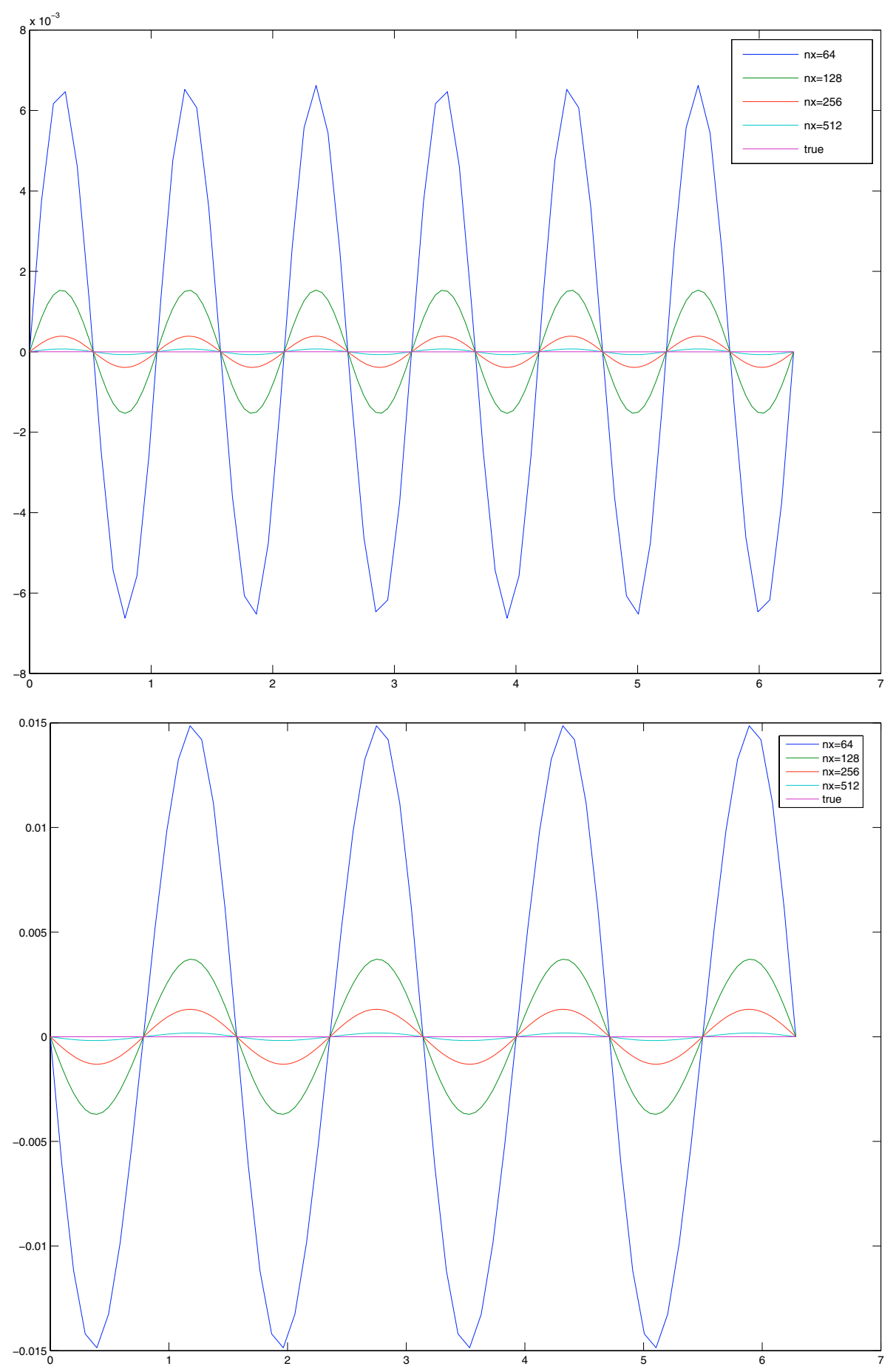

Figure 2. Cross-section of the computed and exact solution: on the top the $u_{1}$ component for $x=\pi$, on the bottom the $u_{2}$ component for $y=\pi$. 

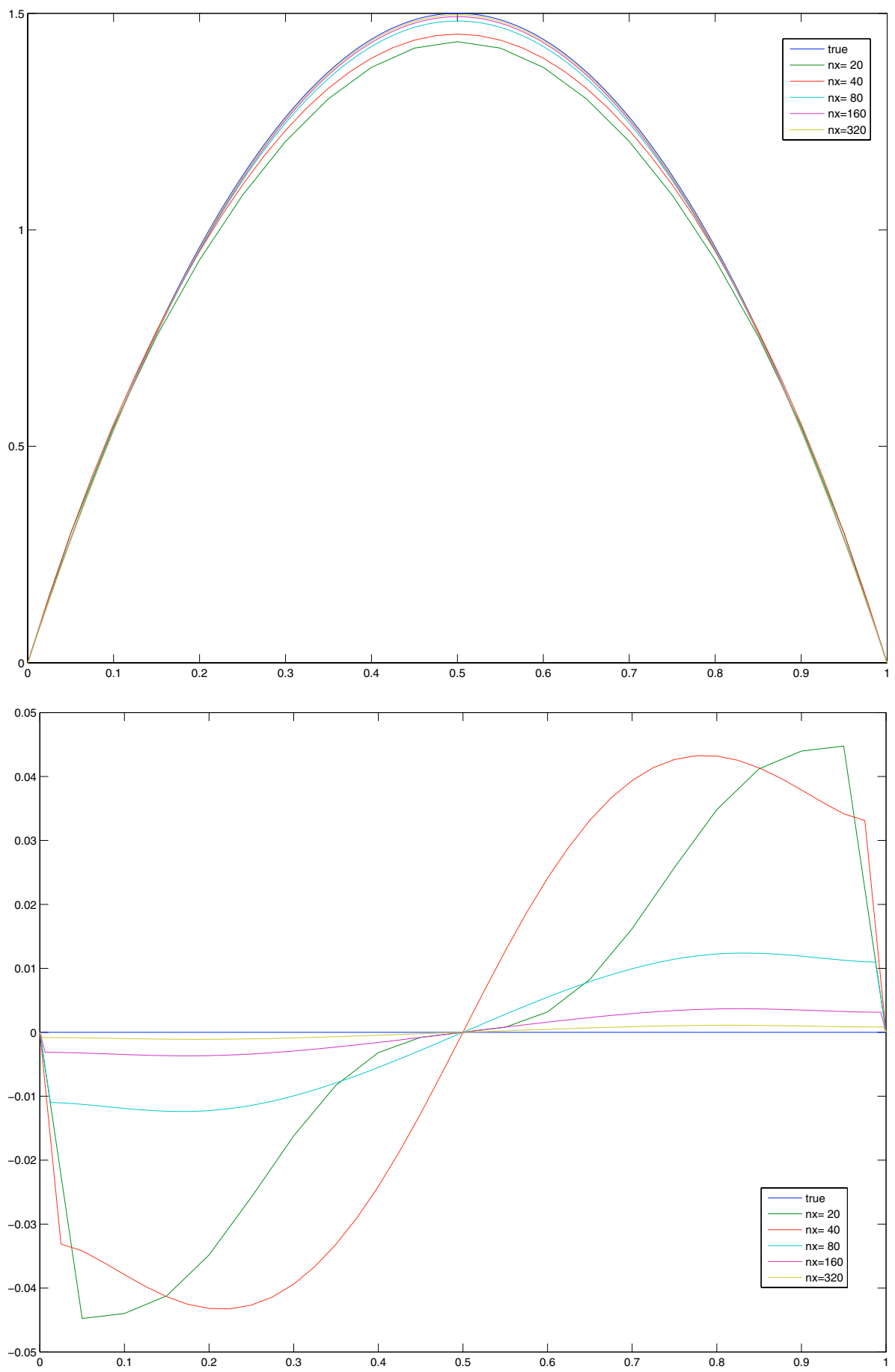

Figure 3. Cross-section of the computed and exact solution for $x=0.5$ : on the top the $u_{1}$ component, on the bottom the $u_{2}$ component. 
The infinitely long channel is modeled by periodic boundary conditions in the $x$ direction; the fixed wall conditions for $\mathbf{u}$ in the $y$ direction are enforced simply by setting $\mathbf{u}=\mathbf{0}$ at the boundary nodes. Choosing a pressure drop $\Delta p=0.12$ and $\nu=0.01$ the theoretical maximal velocity in the channel is $u_{\max }=1.5$.

Again, Table 2 shows accuracy results of the numerical method on the considered test problem, while Figure 3 shows the graphic representation of the velocity profile across the channel for the exact and the computed solution at increasing resolution.

In the results shown in Table 2, the velocity components are approximated almost with a second-order convergence. However, the results of this second test problem suggest the need for a deeper investigation of boundary conditions for our schemes, which will be considered in future works.

\section{Conclusions}

We have presented a kinetic approximation with a discrete set of velocities for the incompressible NavierStokes equations. For this approximation we have given consistency and stability conditions and introduced specific numerical schemes. The tests confirm the nice features of this framework. Clearly, more general schemes in that class could be considered as well as higher order schemes for the kinetic equations, but our main goal in this paper was to show, at least in a preliminary way, the strenght and the flexibility of this approach.

Acknowledgements. The authors thank François Bouchut for many friendly and useful discussions about this work.

\section{REFERENCES}

[1] D. Aregba-Driollet and R. Natalini, Discrete kinetic schemes for multidimensional systems of conservation laws. SIAM J. Numer. Anal. 37 (2000) 1973-2004.

[2] D. Aregba-Driollet, R. Natalini and S. Tang, Explicit diffusive kinetic schemes for nonlinear degenerate parabolic systems. Math. Comp. 73 (2004) 63-94.

[3] M.K. Banda, A. Klar, L. Pareschi and M. Seaid, Compressible and incompressible limits for hyperbolic systems with relaxation. J. Comput. Appl. Math. 168 (2004) 41-52.

[4] S. Bianchini, Hyperbolic limit of the Jin-Xin relaxation model. Comm. Pure Appl. Math. 59 (2006) 688-753.

[5] Y. Brenier, R. Natalini and M. Puel, On a relaxation approximation of the incompressible Navier-Stokes equations. Proc. Amer. Math. Soc. 132 (2004) 1021-1028.

[6] B.M. Boghosian, P.J. Love, P.V. Coveney, I.V. Karlin, S. Succi and J. Yepez, Galilean-invariant Lattice-Boltzmann models with H theorem. Phys. Rev. E 68 (2003) 25103-25106.

[7] F. Bouchut, Construction of BGK models with a family of kinetic entropies for a given system of conservation laws. J. Statist. Phys. 95 (1999) 113-170.

[8] F. Bouchut, Entropy satisfying flux vector splittings and kinetic BGK models. Numer. Math. 94 (2003) 623-672.

[9] F. Bouchut, Nonlinear stability of finite volume methods for hyperbolic conservation laws, and well-balanced schemes for sources, Frontiers in Mathematics series. Birkhäuser (2004).

[10] A.J. Chorin, Numerical solution of the Navier-Stokes equations. Math. Comput. 22 (1968) 745-762.

[11] D. Donatelli and P. Marcati, Convergence of singular limits for multi-D semilinear hyperbolic systems to parabolic systems. Trans. Amer. Math. Soc. 356 (2004) 2093-2121.

[12] W. E and J.G. Liu, Projection method. I. Convergence and numerical boundary layers. SIAM J. Numer. Anal. 32 (1995) 1017-1057; Projection method. II. Godunov-Ryabenki analysis. SIAM J. Numer. Anal. 33 (1996) 1597-1621.

[13] T.Y. Hou and B.T.R. Wetton, Second-order convergence of a projection scheme for the incompressible Navier-Stokes equations with boundaries. SIAM J. Numer. Anal. 30 (1993) 609-629.

[14] M. Junk, Kinetic schemes in the case of low Mach numbers. J. Comput. Phys. 151 (1999) 947-968.

[15] M. Junk and A. Klar, Discretization for the incompressible Navier-Stokes equations based on the Lattice Boltzmann method. SIAM J. Sci. Comp. 22 (2000) 1-19.

[16] M. Junk and W.A. Yong, Rigorous Navier-Stokes limit of the Lattice Boltzmann equation. Asymptot. Anal. 35 (2003) $165-185$.

[17] J. Kim and P. Moin, Application of a fractional-step method to incompressible Navier-Stokes. J. Comput. Phys. 59 (1985) 308-323.

[18] R. Natalini, A discrete kinetic approximation of entropy solutions to multidimensional scalar conservation laws. J. Diff. Equation 148 (1998) 292-317.

[19] R. Natalini and F. Rousset, Convergence of a singular Euler-Poisson approximation of the incompressible Navier-Stokes equations. Proc. Am. Math. Soc. 134 (2006) 2251-2258. 
[20] B. Perthame, Kinetic formulation of conservation laws, Oxford Lecture Series in Mathematics and its Applications 21. Oxford University Press, Oxford (2002).

[21] M. Reider and J. Sterling, Accuracy of discrete velocity BGK models for the simulation of the incompressible Navier-Stokes equations. Comput. Fluids 24 (1995) 459-467.

[22] S. Succi, The Lattice Boltzmann Equation. Oxford University Press, Oxford (2001).

[23] R. Temam, Sur l'approximation de la solution des équations de Navier-Stokes par la méthode des pas fractionnaires. I. Arch. Ration. Mech. Anal. 32 (1969) 135-153; Sur l'approximation de la solution des équations de Navier-Stokes par la méthode des pas fractionnaires. II. Arch. Ration. Mech. Anal. 33 (1969) 377-385.

[24] B.R. Wetton, Analysis of the spatial error for a class of finite difference methods for viscous incompressible flow. SIAM J. Numer. Anal. 34 (1997) 723-755; Error analysis for Chorin's original fully discrete projection method and regularizations in space and time. SIAM J. Numer. Anal. 34 (1997) 1683-1697.

[25] D.A. Wolf-Gladrow, Lattice-gas cellular automata and Lattice Boltzmann models. An introduction, Lecture Notes in Mathematics 1725. Springer-Verlag, Berlin (2000). 